\title{
Crítica a la cultura fósil. La estructura económica como unidad de análisis del cambio cultural paleolítico
}

\author{
A Critique of 'Fossil Culture'. The Economic Structure as the \\ Analytical Unit of Palaeolithic Cultural Change
}

\author{
Policarpo Sánchez Yustos \\ Departamento de Prehistoria y Arqueología. Universidad de Valladolid. Pza. del Campus, s/n. 47011 Valladolid \\ policarpos@hotmail.com
}

Recibido: 20-01-2011

Aceptado: 17-10-2011

\begin{abstract}
ReSUMEN
Actualmente se ha generalizado una interpretación del registro lítico en clave tecnológica y procesual que ha desplazado en parte al enfoque tipológico. Esto ha significado que la variabilidad y el cambio industrial sean contextualizados dentro de los procesos económicos y ambientales en el que se activan las estrategias de talla. Sin embargo, la tipología sigue siendo el único argumento vigente cuando se habla de evolución cultural, pues se continúa empleando la tradicional secuencia evolutiva dividida en "culturas fósiles" definidas a su vez por la agrupación recurrente de "fósiles directores". Asi las cosas, nos parece necesario la creación de un modelo paleocultural que se ajuste a la realidad del registro paleolítico, se libere de la sacralidad del objeto, cuestione la inapelable relación establecida entre morfo-tipo y tradición cultural y suprima el privilegio concedido al registro lítico como marcador absoluto del cambio cultural. Para ello partimos de la asunción de que la evolución cultural va más allá de la variabilidad diacrónica industrial y proponemos la estructura económica como la unidad de análisis del cambio cultural.
\end{abstract}

Palabras Clave: Fósil director. Tipología. Tecnología. Paleolítico.

\begin{abstract}
Nowadays, technological and processual interpretation of the lithic record has been generalized, partly displacing the classical typological approach. Thereby the lithic variability and technological change have started to be contextualized in the economical and environmental processes surrounding the lithic tool production. However, typology is still the only argument when discussing about cultural evolution, since the traditional sequence divided into "fossil cultures", each characterized by distinct and recurrent "guide fossil" assemblages, is widely applied. Thus, in this paper we argue that it is necessary to create a new palaeo-cultural model that more properly suits the Palaeolithic record, getting rid of the sacredness of the object, challenging the direct relationship between morpho-types and cultural traditions and suppressing the lithic record privilege as the only marker of cultural shift. In conclusion, we propose the economic structure as the analytical unit to approach the Palaeolithic cultural change.
\end{abstract}

Key words: Guide fossil. Typology. Technology. Palaeolithic.

Sumario 1. La gran paradoja. 2. El enfoque normativo tipológico. 3. Las primeras alternativas. 4. El enfoque tecnológico. 5. Conclusiones. 6. Discusión. 


\section{La gran paradoja}

Historiográficamente, el foco de interés de gran parte de los paleolitistas se ha centrado en ordenar la variabilidad industrial y la evolución cultural durante la Prehistoria más remota. Hasta tal punto estas dos cuestiones están tan estrechamente relacionadas que en la actualidad la primera sigue siendo empleada para explicar la segunda. De tal forma, el registro lítico se ha convertido en el único exponente para diferenciar entre tradiciones culturales.

La crónica de esta relación se remonta al origen de la arqueología, cuando se trazan los primeros cuadros evolutivos que intentan ordenar y catalogar los restos arqueológicos que se recuperan en las cada vez más numerosas excavaciones. De este modo, aquellos arqueólogos del siglo XIX que se interesan por el origen de la humanidad se afanan en secuenciar cronológica y culturalmente los restos que encuentran en las diversas cuevas y graveras que se exploran, fundamentalmente, en Francia e Inglaterra.

El reconocimiento académico de esta labor detectivesca se produce en 1863, cuando el geólogo británico Charles Lyell certifica la gran antigüedad humana, sitúa su origen en el tiempo geológico y confirma la contemporaneidad de los objetos de piedra tallada y los restos fósiles que desde hacía varias décadas Boucher de Perthes (1847) estaba encontrando en los depósitos del Somme. Dos años más tarde Johan Lubbock utiliza por primera vez la denominación de Paleolítico y Neolítico para subdividir la entonces llamada Edad de la Piedra (Lubbock 1865).

El Paleolítico nace en un cruce de caminos entre diferentes disciplinas, pues de la Geología toma prestado el método estratigráfico, de la $\mathrm{Pa}-$ leontología el concepto de «fósil director» y de la Antropología el interés por ordenar secuencialmente la cultura material. Este último punto es de vital importancia en la medida en que influye decisivamente en el ulterior desarrollo de los estudios paleolíticos.

Los primeros paleolitistas se centran en robustecer la entidad de este periodo mediante su compartimentación en sucesivas etapas, caracterizadas por agrupaciones de fósiles directores ordenados estratigráficamente. Las primeras secuencias se establecen poniendo en relación estratigráfica conjuntos de faunas extintas con series tipológicas de útiles de piedra.

A partir de este caldo de cultivo, durante la primera mitad del siglo XX se define la secuencia crono-cultural que ha llegado hasta nuestros días, en la que cada crono-cultura es establecida a partir de la convergencia espacio-temporal de una serie de rasgos tecno-tipológicos impresos en determinados objetos. En última instancia, este procedimiento de catalogación parte de la premisa de que la morfología de ciertos útiles contiene una serie de ideas y normas que son compartidos por determinadas agrupaciones culturales.

Durante las últimas décadas del siglo XX se confecciona un enfoque de raíz tecnológica como alternativa a los estudios de base tipológica. Bajo este marco, eclosionan diferentes propuestas que ponen de relieve las profundas debilidades que encierra la tipología, a saber: la mera descripción y sistematización de objetos y la custodia de una secuencia evolutiva ordenada en «culturas fósiles» que únicamente existen sobre el papel. Se desmiente así el hecho de que la variabilidad industrial de la que hace gala el registro paleolítico pueda comprimirse en una única secuencia cerra$\mathrm{da}$, definida mediante principios tipológicos.

Actualmente los estudios tecnológicos han suplantado a los tipológicos. Gracias a este giro, la variabilidad industrial se enmarca dentro de los procesos económicos y medioambientales en que se activan las estrategias de gestión de los recursos líticos. Se abre por tanto una puerta a la interpretación procesual y contextual de la de la tecnología, que trasciende su mera lectura en clave cronocultural.

Sin embargo, paradójicamente, todavía es muy habitual ubicar los conjuntos líticos dentro de la secuencia oficial a partir de determinados fósiles directores. Detrás de esta rutina tan extendida subyacen una serie de ideas normativistas cuyos principios han sido supuestamente superados $y$, en el peor de los casos, significa seguir enfatizando el registro lítico como el mejor indicador para diagnosticar el cambio cultural.

De esta forma, se pone de relieve la "gran paradoja" que supone emplear las culturas fósiles para ordenar los conjuntos líticos del Paleolítico cuando supuestamente se reconoce su arbitrariedad y se critican sus principios teórico-metodológicos. Esta circunstancia parece responder a una inercia y falta de reflexión que ha conducido a aceptar una heurística que no se comparte o, como dice G. Clark: "We tend to become consumers of one another's research conclusions" (Clarke 2009: 19).

Con intención de superar esta paradoja, pasamos a resumir el itinerario historiográfico por el que han transitado los estudios paleolíticos. Esta visión retrospectiva, además de aportar una serie de elementos reflexivos imprescindibles en el debate que proponemos, es necesaria para criticar, con argumentos, tanto la secuencia oficial ordenada en culturas fósiles como su heurística. 


\section{El enfoque normativo tipológico}

El primer autor en ordenar (bioestratigráficamente) los materiales paleolíticos fue el francés Edouard Lartet. Tras él son varios los prehistoriadores (como Cartailhac, Reinach, Prestwich, Evans, Falconer, etc.) que ensayan diferentes cuadros evolutivos. Sin embargo, fue el propuesto por Gabriel de Mortillet (1872) el que tuvo más éxito y el que, con algunos retoques y ampliaciones, ha llegado hasta nuestros días.

En un primer momento, Mortillet (1869) utiliza principios bio-estratigráficos para ordenar los restos paleolíticos. No obstante, posteriormente y siguiendo el ejemplo de Lubbock y Worsaae, divide este periodo en función de criterios arqueo-estratigráficos que confieren una importante carga cultural a cada etapa (Mortillet 1872). Éstas son definidas a partir de un número limitado de utensilios (fósiles directores), que son seleccionados subjetivamente en base a la premisa de que la evolución cultural transcurre de las formas simples a las complejas.

$\mathrm{Su}$ ordenación cronológica la establece con ayuda de los principios de la Paleontología estratigráfica. A partir de aquí constituye un axioma de correlación específico como sistema de inferencia: mismos instrumentos igual a misma cronología. De la Geología también toma el sistema de denominación epónima, por lo que las diferentes etapas pasan a rotularse según el nombre del yacimiento más característico: Chelléenne, Acheuléenne, Moustérienne, Solutréenne, Magdalénienne y Tourassienne.

La carga cultural que deposita Mortillet en su secuencia se refuerza poco después, cuando en el marco de las Ciencias Sociales el Evolucionismo es sustituido por el Particularismo histórico, lo que favorece que el registro arqueológico sea leído en clave histórico-cultural. A pesar de que la Arqueología de principios del s. XX rechaza el Evolucionismo, continúa ordenando el registro material en una secuencia de progresión mediante fósiles directores agrupados en listas tipológicas que se emplean para definir culturas. De este modo, los estudios paleolíticos de principios del siglo hacen uso de algunos recursos heurísticos decimonónicos (como son los fósiles-guía culturales y la paleontología estratigráfica) y gracias a su sistematización dejan sentadas las bases del enfoque normativo.

Durante las primeras décadas del siglo XX la impetuosa actividad científica se concentró en revisar y detallar la secuencia establecida por Mortillet. La reforma más significativa la protagoniza el abad francés Henri Breuil (1913), quien además de ampliar la secuencia con nuevas etapas, hace especial hincapié en el método estratigráfico como el procedimiento esencial para fijar su posición relativa en el tiempo, lo que permite hablar con más rigor de crono-culturas. Otra importante contribución de este insigne prehistoriador es su idea de los «philas» culturales paralelos que coexisten durante la primera mitad del Paleolítico. Esta novedad permite plantear la evolución en mosaico y romper definitivamente con los principios filogenéticos unilineales. Asimismo, Breuil establece una clara asociación entre cultura y grupo étnico.

A partir de este marco de actuación, el objetivo que se plantean los paleolitistas de la primera mitad del siglo XX es excavar -cuando más cuevas y abrigos mejor-, para dotar de más precisión y oficializar definitivamente la secuencia de Mortillet. A finales de la década de los treinta este objetivo se ha cumplido, pues para toda Europa partiendo de los eventos glaciares se ha establecido un cuadro cultural con su correspondiente correlación cronológica.

Este ímpetu en los trabajos de campo se traduce en la presentación de múltiples secuencias locales y regionales. De esta forma, contrariamente a los fundamentos particularistas que conlleva la propuesta del abate Breuil, los paleolitistas del momento intentan encajar sus colecciones dentro de la secuencia oficial diseñada a partir de las colecciones-tipo recuperadas en los yacimientos-tipo franceses.

La falta de homogeneidad que muestra el registro, unida a los estrechos márgenes que encierra la secuencia oficial, exige a los arqueólogos forzar sus sitios para encajarlos dentro de la misma. También impone la creación de una serie de recombinaciones terminológicas imposibles y la consiguiente aparición de una panoplia de nuevos conceptos (fase, edad, faceta, complejo cultural, tradición cultural, etc.), que si bien es cierto que alivian la tensión existente entre el Evolucionismo y el Particularismo, conducen a una serie de importantes imprecisiones cuyo resultado final es una confusión semántica y el total vaciado del propio concepto de cultura. Este galimatías se intenta solucionar en la década de los treinta parcelando el periodo Paleolítico en tres fases o periodos (inferior, medio y superior), que carecen de una carga cultural explícita ya que su función es almacenar y, a la par, ordenar en el tiempo el espeso conglomerado de tradiciones crono-culturales regionales registradas.

La distinción del Paleolítico superior con respecto a las otras fases resulta sencilla, pues la elevada estandarización de sus fósiles directores permite una fácil adscripción dentro de la secuencia oficial. Sin embargo, desde el principio, la distinción entre Paleolítico inferior y medio se muestra más complicada. Para esta demarcación Breuil recurre al viejo axioma decimonónico por el cual el progreso se opera de lo simple a lo complejo. Así, para catalogar los conjuntos se emplean criterios estilísticos, 
relacionados, por ejemplo, con la simetría y regularización de filos de ciertos útiles como el bifaz. No obstante, debe quedar claro que estas "observaciones" no son inferidas estratigráficamente, condición sine qua non de su propia metodología.

En este delicado momento aparecen en escena François Bordes y Denise de Sonneville-Bordes, quienes formulan una metodología sistemática con la que pretenden dejar atrás ciertas ambigüedades y vaguedades (Bordes 1961; Sonneville-Bordes y Perrot 1954-1955). En un primer momento, F. Bordes (1950) presenta una teoría explicativa que trata de organizar el registro del Paleolítico inferior y medio a través de dos componentes morfo-tipológicos principales: la morfología de los bifaces y la evolución del método Levallois, aunque también tiene en cuenta los útiles sobre lasca y el facetado de las mismas. A partir de la articulación de estos criterios se diferencia el Achelense del Musteriense, de la misma forma que se distingue tres fases dentro del Achelense (inferior, medio y superior). Poco después, siguiendo esta clase de pautas, clasifica las industrias musterienses en 12 grupos teóricos, aunque según reconoce el mismo Bordes algunos eran desconocidos en la práctica (Bordes 1953).

A partir de estos trabajos germinales, F. Bordes (1961) desarrolla un sistema tipológico cerrado para el Paleolítico inferior y medio, mientras que Sonneville-Bordes junto con Perrot (1954-1955) hace lo propio para el Paleolítico superior. Ambos modelos están acompañados por una serie de técnicas destinadas a describir cuantitativamente las series líticas, por lo que para su atribución cultural se tienen en cuenta "todos" los implementos retocados y otras formas significativas, así como sus frecuencias.

En concreto, la mecánica del método que aplica F. Bordes para las series musterienses se inicia con el conteo de una serie de atributos, individualizados o agrupados en asociaciones recurrentes (tipos) y su consiguiente clasificación en función de la lista tipológica que establece. Seguidamente, se calcula sus índices y se traza un gráfico acumulativo donde se refleja la frecuencia de los diferentes tipos. El aparato estadístico que se despliega en estas curvas de frecuencias acumulativas está destinado a representar gráficamente el conjunto lítico (a partir de 100 útiles). En función de la gráfica que dibujan los porcentajes de los distintos tipos, el conjunto es clasificado dentro de las categorías culturales establecidas: Musteriense de tradición achelense, Musteriense típico, Musteriense con denticulados y Musteriense charentiense, este último subdividido en Quina y Ferrassie.

Realmente esta reforma metodológica supuso un salto cualitativo (a nivel empírico) con respecto a lo que se estaba haciendo hasta el momento, pero no fue acompañada de un cuerpo teórico original. Esta circunstancia condujo a Bordes a sumarse a las interpretaciones normativas de la cultura, por lo que las diferencias tipológicas entre los conjuntos musterienses (philae paralelos en el sentido de Breuil) las presenta como el reflejo de la identidad cultural de los diversos grupos o tribus que los han fabricado. Por otro lado, la carga ideográfica que conlleva el normativismo arrastra a Bordes a la obsesión por el útil y al olvido de la técnica. Está descompensación la justifica asegurando que la técnica no sirve para reconstruir la historia cultural de los pueblos, ya que son los útiles lo que caracteriza a cada época (Bordes 1947: 28).

Sea como fuere, el aparato heurístico que condensa este enfoque, que alcanza su cénit con la figura de F. Bordes, se caracteriza por entender la cultura como un cuerpo de ideas, valores y creencias compartidas. De tal modo, la tarea del arqueólogo radica en destilar de los productos culturales los conceptos normativos existentes en las mentes; esto es, descubrir inductivamente las ideas que gobernaron la producción de objetos, pues éstos son elaborados a través de plantillas mentales. Según esta premisa, la evolución material y cultural durante el Paleolítico se ordena mediante una lista tipológica de utensilios, cuyo cambio y variabilidad se lee en clave ideográfica y, por ende, cultural o biológica. Sin embargo, después de la conocidísima réplica de los Binford acerca de la variabilidad del Musteriense (Binford y Binford 1966; Binford 1973), Bordes parece enmascarar su normativismo cultural al sugerir que lo que existe es un amplio margen de variación en una serie de conjuntos que denominamos musterienses por conveniencia (Bordes 1977: 38).

\section{Las primeras alternativas}

\subsection{La alternativa francesa}

En paralelo al tipologismo cultural desarrollado por el matrimonio Bordes, G. Laplace comienza a definir las bases de su tipología analítica, al tiempo que defiende la sugerente idea de que las industrias evolucionan como resultado de un conflicto de adaptación del ser humano a su medio ambiente (Laplace 1956, 1958). Para este autor, la evolución industrial se lleva a cabo en focos independientes y está influida por las culturas limítrofes, siguiendo cambios cuantitativos que al llegar a cierta intensidad y tensión provocan un cambio mutacional, siempre y cuando no desaparezca por su inadaptación, con lo que aparecen nuevas formas articuladas entre sí de modo arborescente. 
Su propuesta analítica definitiva se materializa en su obra Tipología Analítica y Estructural (Laplace 1972). Esta novedosa orientación metodológica se fundamenta en un sistema de análisis que jerarquiza y estructura las características morfo-técnicas de los productos formateados mediante retoque. Para ello, Laplace se centra en una serie de caracteres significativos que permiten identificar tipos morfotécnicos, al tiempo que emplea una estadística inferencial adaptada a su tratamiento analítico, que acompaña con una serie de descripciones ordenadas mediante un lenguaje estandarizado.

También en paralelo al enfoque tipologista, se gesta el concepto de cadena operativa, cuyo caldo de cultivo es la Etnología estructuralista francesa de mediados de siglo, momento en que Mauss (1947) subraya la importancia de analizar las diferentes etapas de la fabricación de los objetos. Poco tiempo después, Maget (1953) habla de "cadena de fabricación" y sugiere la descomposición de las actividades técnicas hasta el nivel de gestos elementales. El primero en incorporar esta idea a la Arqueología es Leroi-Gourhan (1964), quien emplea el término de "cadenas de gestos estereotipados" como el elemento primordial de la supervivencia humana, aunque tendrán que pasar algunos años para que se generalice su uso.

$\mathrm{Su}$ desarrollo conceptual definitivo e introducción en el campo de las secuencias líticas se debe a Lemonnier (1976) y Cresswell (1976). Este hecho marca una profunda separación con la Arqueología histórico-cultural y sobre todo supone enfatizar la secuencia técnica restando importancia al producto final para concedérsela a la sucesión secuenciada y encadenada de su producción. Así, tal y como señala J. Pelegrin $(1985,1990)$, a partir de los ochenta aparece una nueva generación de paleolitistas que dejan der ver los útiles de piedra como meros ítems culturales y pasan a interesarse por su dimensión técnica, económica, social y cultural.

\subsection{La alternativa anglosajona}

La Nueva Arqueología organiza una contundente ofensiva contra el enfoque normativo de la cultura, pues defiende una perspectiva dinámica, procesual, sistémica, materialista, ambiental y conductual de la cultura, que subraya el proceso de adaptación de la sociedad al medioambiente (Binford 1965; Flannery 1967; Struever 1971; Isaac 1972). De esta manera, se prefiere explicar el cambio socio-cultural y la variabilidad instrumental en términos ecológicos, adaptativos y funcionales.

Con toda seguridad, L. Binford (Binford y Binford 1966, 1969; Binford 1972, 1973) protagoniza la crítica más conocida, que no la más acertada o mejor justificada (Vega Toscano 2001: 203), a la interpretación de las facies musterienses establecidas por Bordes. Este arqueólogo norteamericano tras aplicar una serie de técnicas estadísticas de análisis multivariante, empleando para ello la metodología de Bordes de las industrias examinadas, concluye que la variabilidad del musteriense responde a una distribución espacial desigual de un conjunto de utensilios empleados en diversas actividades. Sin embargo, esta hipótesis sufre un fuerte revés en el momento en que se analizan las huellas de uso de las herramientas líticas y se demuestra la versatilidad del instrumental musteriense (Anderson 1981; Beyries 1987).

En cualquier caso, desde el procesualismo se pone en cuestionamiento la propia lógica normativa de la escuela tipológica, tanto al dudar de la sobreentendida correspondencia entre tipo y patrón mental, como al subrayar así la gran dificultad que entraña su justificación demostrativa. Al mismo tiempo, se rechaza la idea de que el cambio cultural sea entendido como un mero accidente histórico fruto de la migración o difusión, mientras que por otro lado se interesan por los procesos de producción tecnológicos en el marco de la ecología y funcionalidad de las ocupaciones.

En definitiva, los primeros arqueólogos procesuales (entre los que destacan L. Binford, D.L Clarke y G. Isaac) argumentan la variabilidad industrial a través de diferentes causas coyunturales, que no hacen sino poner de manifiesto los diferentes factores que intervienen en los procesos tecnológicos y en la forma final de los objetos. Todo ello contribuye a que la variabilidad deje de ser leída de forma exclusiva en clave cultural. Sin embargo, paradójicamente continúan empleando la secuencia normativista para catalogar los conjuntos.

Esta última tendencia es rota por Clark (1969: 39ss.) al formular una propuesta alternativa a la secuencia oficial. Este autor propone un esquema evolutivo lineal en el que condensa y ordena cronológicamente los principales cambios que se operan en la industria lítica prehistórica. A partir de pautas globales ajustadas a un proceso acumulativo de cambio, sintetiza toda la variabilidad diacrónica en cinco agrupaciones tecnológicas homogéneas denominadas Modos técnicos. En cada uno se acumulan los esquemas técnicos heredados de las etapas anteriores, junto con aquellas novedades que marcan el cambio. Precisamente, son estos nuevos elementos tecnológicos (como el bifaz o la técnica Levallois) los que establecen la diferencia con respecto a los Modos anteriores y, por tanto, se emplean como marcadores tecno-tipológicos absolutos que permiten catalogar un conjunto industrial dentro de esta secuencia. 
Entre los inconvenientes de este sistema sobresale su vocación excesivamente sintética y unilineal, lo que dificulta su ajuste a la variabilidad industrial y acarrea ciertos problemas de imprecisión cronológica (Bar Yosef y Belfer Cohen 2001; Díez Martín 2004; Gamble 2001; Villa 2001). Por ejemplo, en el registro arqueológico del viejo continente, en numerosas ocasiones la presencia de aquellos elementos tecnológicos que son empleados para marcar el cambio de Modo tienen una presencia intermitente durante largos periodos de tiempo. De hecho, su presencia o ausencia no tiene por qué estar unívocamente relacionada con una secuencia cronológica donde la evolución cultural es ordenada de forma lineal y progresiva.

\subsection{La alternativa ibérica}

A principios de los ochenta, en el noreste de la Península Ibérica se empieza a desarrollar una propuesta teórico-metodológica alternativa al paradigma tipológico y denominada Sistema Lógico Analítico (Carbonell et al. 1983). Recoge las principales propuestas metodológicas que a finales de los setenta pululan en los ambientes reformistas franceses y en gran media se empapa de la Tipología Analítica y Estructural de Laplace (1972), al tiempo que en sus fundamentos teóricos se combina la dialéctica del materialismo histórico con el funcionalismo ambiental.

Desde este singular punto de partida se pretende integrar el medio natural con la realidad social (Carbonell et al. 1983; 1992; Mora et al. 1991). Para ello se considera que el cambio cultural se opera desde la propia dinámica social, enmarcada por las estrategias de gestión de los recursos que, al mismo tiempo, están condicionadas por la experiencia acumulada y transmitida. Consiguientemente, la organización social no se corresponde de forma exclusiva con la estructura económica, en tanto que las ideas o la propia conciencia germinan de la experiencia acumulada por la sociedad. En suma, los cambios en el sistema tecno-económico son consecuencia de la dialéctica sociedad-naturaleza.

Se aboga de este modo por un conocimiento dinámico de los procesos por los que atraviesa un objeto cuando es sometido a intervención. Así, la dialéctica surgida entre los factores sociales y ecológicos delinea totalmente las estructuras de los Sistemas Operativos Técnicos (terminología propia del SLA) y a partir de la relación que el grupo humano establece con el entorno natural se implantan las estructuras técnicas.

También el SLA ha pretendido explicar y ordenar el cambio y transformación morfológica de los instrumentos líticos con su "Teoría de la transferencia morfotécnico-funcional-potencial" (Carbonell y Mora 1986; Carbonell y Sala 1989; Carbonell 1990; Carbonell et al. 1992). Su punto de partida es la concepción del útil como una estructura compuesta por tres elementos jerarquizados (morfotécnico, morfopotencial y morfofuncional), cada uno de los cuales marca un periodo: Biomorfofuncional $(2,5-$ 1,5 Ma.), caracterizado por una producción inmediata de los objetos para un uso expeditivo; Biomorfotécnico (1,6 Ma.-30 Ka.), cuando se generaliza la estandarización de objetos con morfologías precisas; y por último, el Biomorfopotencial (30-6 Ka.), periodo en el que se crean instrumentos con los que se maximiza la capacidad potencial de intervención sobre la materia.

En realidad, este es un modelo muy elemental de periodización de la tecnología paleolítica que tropieza con la misma piedra que el enfoque tipológico al que critica, a saber: el papel hegemónico que se concede a una parte del registro lítico (los objetos retocados y determinados tipos de lascas) como vector de ordenación cronológica de los procesos morfotécnicos. Tal vez por esta razón, y dado que este modelo únicamente sirve para la transformación morfológica de los instrumentos, sus creadores, aparte de explicarlo teóricamente en diferentes ocasiones, no acostumbran a emplearlo como marco donde adscribir los conjuntos líticos que estudian, tarea para la que prefieren utilizar los Modos tecnológicos (ej. Carbonell et al. 1999; Carbonell et al. 2001; 2009).

\section{El enfoque tecnológico}

A partir de estos focos reformistas, en las últimas décadas se ha consolidado una investigación de corte tecnológico que enfatiza las secuencias de talla y su estrecha relación con el contexto económico que dirige su producción.

En torno a esta última cuestión converge la escuela francesa con la anglosajona (p. ej., citando únicamente las referencias primeras y más significativas: Geneste 1985, 1988a, 1988b, 1989, 1991a y 1991b; Meignen 1988; Otte 1990; Tavoso 1984; Turq 1986, 1989), aunque la primera también desarrolla una línea de investigación propia que se concentra en los aspectos conceptuales y técnicos (p. ej. Boëda 1988, 1991, 1994; Bourguignon 1997, 1999; Geneste, 1991a; Inizian et al. 1995; Pelegrin 1985, 1990, 1995; Tixier 1982). Por su parte, los autores anglosajones siguen la estela abierta por L. Binford y G. Isaac (este último más interesado en las primeras industrias africanas) y sobre todo se interesan en establecer vínculos entre los atributos 
morfotécnicos de ciertos objetos y los factores económicos y ecológicos (ej. Dibble 1988, 1989, 1991, 1995; Dibble y Rolland 1990, 1992; Dibble et al. 1995; Hayden 1989; Kuhn 1991, 1992, 1993, 1995; Rolland y Dibble 1990).

El salto cualitativo que significa este enfoque dentro de los estudios paleolíticos responde al interés que muestra por la complejidad conceptual y operativa de los procesos tecnológicos y su intención de encuadrar dichos procesos dentro de su contexto de producción. De esta forma consigue ampliar los horizontes disciplinares, en la medida en que formula una amplia gama de preguntas y respuestas que profundizan en los diferentes dominios que conforman la variabilidad intraespecífica de los conjuntos líticos.

Por esta última razón, y también por una cuestión de espacio y conscientes de que desarrollar todas las líneas de investigación seguidas en los estudios de corte tecnológico bien merece un trabajo aparte, queremos detenernos brevemente en su dimensión económica. Precisamente, desde aquí se estructura una interpretación alternativa y crítica a la ofrecida por el enfoque tipológico en relación a la variabilidad en las industrias paleolíticas.

Aproximarse a esta cuestión, en primer lugar, nos sitúa frente al nudo gordiano del enfoque tecnológico, a saber: qué papel juega el ambiente en los procesos tecnológicos. La respuesta, que no es sencilla, no se ha buscado en el determinismo ambiental, sino más bien en la Ecología humana, que sostiene que los sistemas naturales y socio-culturales son dinámicos $\mathrm{y}$, por tanto, extremamente sensibles a las variaciones de las condiciones iniciales, lo que conduce a ambos sistemas hacia una constante auto-organización y coevolución (Hawley 1991). Por otro lado, hemos de ser conscientes de que la toma de decisiones de una determinada comunidad humana ante cambios ambientales más o menos drásticos también está determinada por su sinergia socio-cultural. Esta circunstancia explica por qué ante las mismas condiciones ambientales los grupos humanos desarrollan diferentes respuestas adaptativas. En consecuencia, para valorar el papel que juega el ambiente en los procesos tecnológicos, además de conocer lo mejor posible el medio natural, es necesario adentrarse en las relaciones que un grupo humano mantiene con el ecosistema en el que vive y del que forma parte.

Precisamente, esto es lo que hacen las investigaciones que se adentran en la dimensión económica de la tecnología y sondean los factores externos que influyen en las estrategias de talla. Entre éstos, tradicionalmente han sobresalido los estudios territoriales que, desde los trabajos pioneros de J. Steward $(1950,1955)$, han explicado cómo las estrategias de gestión de los recursos líticos mantienen una estrecha relación con los patrones de asentamiento (p. ej. Willey, 1953; Binford y Binford 1966; Isaac, 1981; Marks 1988; Magne 1989; Lieberman 1993; Kuhn 1995).

Siguiendo esta línea argumental, también se ha demostrado cómo la transformación morfométrica de algunos objetos está vinculada tanto con su transporte como con el tipo, intensidad y duración de las actividades en las que participa (p. ej. Dibble 1988, 1989, 1991, 1995; Dibble y Rolland 1990, 1992; Dibble et al. 1995; Geneste 1985, 1988a, 1988b y 1990; Hayden 1989; Kuhn 1991, 1992, 1993, 1995; Meignen 1988; Otte 1990; Rolland y Dibble 1990).

Estas investigaciones se han completado con otras que profundizan en cuestiones relacionadas con la optimización, accesibilidad y características físicas de los recursos líticos (Geneste 1988a, 1988b, 1989, 1991b; Tavoso 1984; Turq 1986, 1989). En ellas se alcanzan importantes conclusiones, como por ejemplo que los materiales más próximos al sitio de ocupación son introducidos en formas poco elaboradas y sus cadenas operativas tienen un desarrollo netamente local. En cambio, conforme aumenta la distancia al área de captación de los materiales introducidos en el sitio ocupado, éstos suelen ser de mejor calidad, se reservan para las estrategias de talla más exigentes técnicamente, están intensamente aprovechados y sus cadenas operativas aparecen más fragmentadas.

En definitiva, aunque únicamente hemos enumerado los principales campos de interés en los que se centra la dimensión operativa del enfoque tecnológico, es suficiente para percibir la interpretación orgánica que hace del registro lítico y los argumentos que emplea para desplazar a un segundo plano la tipología. Sin embargo, es una "gran paradoja" que en el seno de este enfoque se mantengan las categorías tipológico-culturales para hablar de evolución cultural cuando precisamente los estudios tecnológicos han demostrado su arbitrariedad.

\section{Conclusiones}

A lo largo del texto hemos tenido la oportunidad de comprobar la ineficacia heurística que presentan las culturas fósiles para explicar la variabilidad industrial y el cambio cultural. Detrás de su rutinaria aplicación se esconde una anacrónica actividad catalogadora que sacraliza el objeto y favorece una sensación de estar constantemente ante transiciones culturales. La miopía endémica existente en la ordenación del registro lítico en crono-culturas -o Modos técnicos- induce a suponer que se está ante un fenómeno transicional cuando en realidad 
lo que existe es una lógica presencia intermitente de los fósiles directores que se emplean para ordenar dichas secuencias. De hecho, la propia noción de "industria transicional" es el resultado de una construcción tipológica (Straus 2007).

Esta sensación se amplifica con el problema que entraña establecer los límites entre periodos. Por ejemplo, la distinción entre Paleolítico inferior y medio se sigue mostrando muy forzada (p. ej. Monnier 2006; Chazan 2009), lo que ha propiciado que cada vez más investigadores seamos partidarios de unificar en un solo grupo y bajo un mismo término (Paleolítico antiguo) las industrias de ambas etapas (p. ej. Bar-Yosef 1982: 32; Gamble1990: 134; Boëda 1991: 37; Stringer y Gamble 1996: 154; Vega Toscano 2003: 71; Sánchez Yustos 2009).

En este caso concreto, la dificultad que implica delimitar la frontera entre ambos periodos radica en que la técnica Levallois es el principal marcador que rige ese cambio. Sin embargo, en el registro empírico este método de talla no supone una nítida frontera cronológica o tecnológica (Villa 2009: 269).

Por otra parte, el copioso caudal terminológico derivado de la secuencia oficial ha generado trasvases conceptuales, fruto del manoseo y erosión que han sufrido los diferentes términos al ir saltando de escuela en escuela. Tras tanto vaivén se ha terminado por olvidar -o dejar de importar- en cuáles ésta puesto el acento cultural. Esta circunstancia ha conducido a un preocupante vaciado y uso sesgado del concepto de cultura.

Paradójicamente este vaciado se ha intensificado después de que el enfoque normativo haya pasado a un segundo plano. Los estudios tecnológicos han sido capaces de demostrar que la visión idealizada del concepto de cultura es imposible de acomodar a un registro que se caracteriza por una gran diversidad y multiplicidad de formas que no siempre responden a una lógica temporal, pero han sido incapaces de proponer una alternativa en este sentido. Esta importante laguna, explicada en parte por la inercia y una actitud poco reflexiva, se ha disimulado al aprovechar el valor sintético y descriptivo que muestran las clásicas categorías tipo-culturales.

Un ejemplo esclarecedor de esta enorme confusión es la equiparación conceptual que han sufrido términos como Achelense, Paleolítico inferior y Modo 2; aunque también es bastante común referirse al Achelense como un tecnocomplejo. En rigor, cuando se habla de Achelense nos referimos a una crono-cultura, mientras que Paleolítico inferior o Modo 2 son términos que simplemente hacen alusión a un estadio de una secuencia evolutiva trazada desde presupuestos tecno-tipológicos. Por otra parte, según la definición que da Clarke de "tecno- complejo" (un grupo de culturas que comparten un conjunto de objetos y técnicas que actúan como respuesta ampliamente difundida ante una serie de factores comunes de orden económico [Clarke 1984: 295]), no es correcto referirse al Achelense como un tecnocomplejo; en este caso, lo más acertado sería calificar de tecnocomplejo al Paleolítico inferior.

Por último, sin voluntad de detenernos en la construcción histórica del concepto de cultura en las Ciencias Sociales, en términos generales la cultura puede ser entendida como un fenómeno plural, complejo y orgánico, contrapuesto a la naturaleza. Por esta razón, nos parece un planteamiento excesivamente simplista acercarnos a este fenómeno prestando atención únicamente a ciertos objetos de piedra o en el mejor de los casos a los procesos tecnológicos. En nuestra opinión, esta actitud ha favorecido que el registro lítico se establezca como el marcador absoluto de las tradiciones culturales y, por ende, del cambio cultural.

Nos parece necesario que en los estudios paleolíticos la evolución cultural vaya más allá de la mera variabilidad industrial. El primer paso en esta dirección es abolir tanto el afán catalogador como la inapelable relación que se ha establecido entre morfo-tipo y tradición cultural, así como suprimir el privilegio concedido al registro lítico como marcador cultural. El segundo sería reflexionar disciplinarmente sobre la necesidad de definir un nuevo modelo cultural para el Paleolítico.

\section{Discusión}

Llegados a este punto, ante la necesidad de adecuar el modelo a la realidad que muestra el registro, la primera pregunta que debemos responder es si la cultura puede seguir siendo una unidad de análisis dentro de los estudios paleolíticos. Evidentemente, la respuesta pasa por reconocer en primer lugar cuál es el tipo de información al que tenemos acceso.

El registro paleolítico fundamentalmente proporciona información económica y subsistencial (Clark 1952; Domínguez Rodrigo 2008); esto es, información sobre los patrones comportamentales destinados a la administración del oikos (oikonomía), que en los grupos no productores hace alusión al lugar físico de ocupación, al grupo asentado, a los recursos explotados y al territorio donde éstos se localizan (Sánchez Yustos 2009: 10).

Esta circunstancia restringe nuestras posibilidades de acercarnos de un modo directo a las múltiples esferas que definen a una cultura. En cierta medida, esta deficiencia no es tan determinante si tenemos en cuenta que nos movemos en un periodo en el que muchas de esas esferas todavía no for- 
man parte, o lo hacen de un modo muy tímido -casi imperceptible-, de las múltiples dimensiones que realmente conforman la cultura. En cualquier caso, la estructura económica -mejor dicho la estructura oikonómica- se convierte en el mejor y único camino para acceder al sistema socio-cultural.

En consecuencia, aunque somos conscientes de que en el cambio cultural pueden estar implicados muchos más factores que los estrictamente económicos (biológicos, ambientales, procesos culturales internos o procesos de aculturación causados por migración interregional e interacción intergrupal), nos vemos obligados a abordar la cuestión de la evolución cultural desde una óptica más científica y menos historicista, y asumir en buena parte de nuestras interpretaciones un determinismo infraestructural.

Esta enfatización de la infraestructura se traduce en la necesidad de contextualizar temporal y espacialmente todos los comportamientos que conforman la estructura económica, a saber: las modalidades de apresado y procesado de los recursos bióticos, las modalidades de gestión de los recursos líticos, la estructuración del espacio habitado y del territorio explotado, el empleo del fuego, etc.

De esta forma, el comportamiento tecnológico se presenta como una hebra más del gran tejido económico. Esto no impide que reconozcamos el componente cultural que en general contiene la tecnología lítica y, en particular, la morfología final conferida a ciertos objetos. Sin embargo, dudamos de hasta qué punto se acoplan los tiempos de las estructuras tecnológicas y culturales y si los denominados episodios de transición tecnológica están acompañados o espoleados por fenómenos de transición cultural.

Pretendemos que uno de los puntos de anclaje hacia la construcción de un nuevo modelo paleo-cultural sea la contextualización temporal y espacial de los diferentes comportamientos subsistenciales que conforman la estructura económica. Y para evitar la falta de concreción que supone todo modelo generalista, centrado en grandes tendencias excesivamente abstractas y alejadas de la realidad que refleja el registro (p. ej. Groenen 1994; Monnier 2006; y los artículos de Harrold, Soffer, Strauss, Gowlett o Clark en Camps y Chauhan 2009), proponemos que el marco espacial de esta contextualización sea un ecosistema determinado. De este modo, queremos abordar la evolución cultural desde un radio de acción regional y con una perspectiva territorial que propicie la contextualización de la sociedad con el medio natural que habita. Precisamente, ya que entre los grupos humanos no productores los patrones de movilidad y ocupación del territorio son la correa de distribución de las actividades subsistenciales, como ya propuso J. Steward (1955), resul- tan un perfecto indicador para detectar los cambios culturales.

Otro camino para alejarse de la excesiva generalización consiste en dejar de asociar la evolución con la idea de progreso, pues exige secuenciar linealmente ciclos de crecimiento mediante discontinuidades excesivamente abstractas y generalistas. Por el contrario, resulta más actual y realista entender el cambio cultural como un continuum cuyo hilo conductor es el aumento de la complejidad propiciado por la intervención de más elementos en el sistema socio-cultural, que se transmiten a lo largo de cadenas de funciones en un proceso que se extiende en el tiempo. De esta forma, la evolución cultural se puede presentar como un proceso multifactorial complejo que va más allá de la variabilidad industrial y de las clásicas categorías tipo-culturales.

Esta asunción nos hace rehuir de cualquier secuencia filogenética que intente ordenar la cultura en grandes estadios evolutivos. En cambio, nos aproxima a una estructura abierta formada por múltiples modelos, cada uno adscrito a un ecosistema o territorio determinado, en donde en relación diacrónica se asocian las estructuras económicas observadas en cada caso, que a su vez son jalonadas con aquellas transformaciones que cualitativamente contribuyen a complejizar las relaciones que las sociedades mantienen con el medio ambiente donde se desarrollan.

Volviendo a la pregunta que preside esta discusión, después de todo lo expuesto, consideramos que la cultura no puede seguir siendo una unidad analítica dentro de los estudios paleolíticos. El carácter más teórico qué practico de este concepto, así como la complejidad y multidimensionalidad que engloba, es incompatible y difícil de adaptar a las características y limitaciones que presenta el registro paleolítico.

En suma, abogamos por la estructura económica como la unidad analítica adecuada para abordar la cuestión del cambio cultural. Las razones de peso que avalan nuestro posicionamiento son que la estructura económica no es un concepto exclusivamente teórico; su forma queda fosilizada tanto en el registro paleolítico como en su distribución territorial y por ello tenemos un acceso directo a su materialidad; y, lo más importante, es la puerta que nos conduce al sistema socio-cultural, puesto que, en última estancia, un cambio en la estructura económica nos indica un cambio en el sistema sociocultural. 


\section{REFERENCIAS BIBLIOGRÁFICAS}

Anderson, P.C. (1981): Contribution metodologique á l'analyse des microtraces d'utilisation sur les outils préhistoriques. 2 vols., Thése 3me. Cycle, Univ. Bordeaux I.

BAR Yosef, O. (1982): Some remarks on the nature of transition in Prehistory. The transition from Lower to Middle Palaeolithic and the Modern Man (A. Ronen, ed.), Cambridge: 29-33.

Bar Yosef, O; Belfer Cohen, A. (2001): From Africa to Euroasia: early dispersals. Quaternary International, 75: $19-28$.

Beyries, S. (1987): Variabilité de l'industrie lithique au Moustérien. Approche fonctionnelle sur quelques gisements français. B.A.R. Int. Series, 238, Oxford.

Binford, L.R. (1965): Archaeological systematic and the study of culture process. American Antiquity, 31 : $203-$ 210.

Binford, L.R. (1972): Comments on evolution. An archaeological perspective. (L.R. Binford, ed.), Duckworth, Londres: 105-113.

BINFORD, L.R. (1973): Interassemblage variability: the Mousterian and the functional argument. The Explanation of Culture Change (C. Renfrew, ed.), Duckworth, Londres: 227-254.

BINFORD, L.R..; BINFORD, S.R. (1966): A preliminary analysis of functional variability in the Musterian Levallois facies. American Anthopologist, 68(2): 238-295

Binford, L.R..; Binford, S.R. (1969). Stone tools and human behavior. Scientific American, 220 (4): 70-84.

BoËDA, E. (1988): Le concept Levallois et évaluation de son champ d'application. L'Homme de Néandertal, 4: $13-26$.

BoËDA, E. (1991): Approche de la variabilité des systèmes de production lithique des industries du Paléolitique inférieur et moyen: chronique d'une variabilité attendue. Techniques et culture, 17-18: 37-79.

BoËDA, E. (1994): Le concept Levallois: variabilité des méthodes. CNRS, París.

Bordes, F. (1947): Étude comparative des différentes techniques de taille du silex et des roches dures. L'Anthropologie, 51: 1-29.

Bordes, F. (1950): L'Évolution buissonnante des industries en Europe occidentale. Considérations théoriques sur le Paléolithique ancien et moyen. L'Anthropologie, 54: 393-404.

Bordes, F. (1953): Levalloisien et Moustérien. Bulletin de la Société Préhistorique Française, 50 (4): $226-235$

Bordes, F. (1961): Typologie du Paléolithique ancien et moyen. CNRS, Burdeos.

Bordes, F. (1977): Time and space limits of the Mousterian. Stone tools as cultural markers (R. V. Wright, ed.), Australian Institute of Aboriginal Studies, Camberra.

Boucher de Perthes, J. (1847): Antiquités celtiques et antédiluviennes. Treuttel \& Wurtz, París.

Bourguignon, L. (1997): Le moustérien de Type Quina: Nouvelle définition d'une entité technique. Tesis Doctoral inédita, Université Paris X, París.

Bourguignon, L. (1999): La Conception de Débitage Quina. Quaternaria Nova, IV: 149-169.

Breuil, H. (1913): Les subdivisions du Paléolithique supérieur et leur signification. Congrès International d'Anthropologie et d'Archéologie préhistoriques, Comptes Rendus, 14ème session, Genève, 1912 : 5-78.

Carbonell, E. (1990): Desarrollo tecnológico y desarrollo humano en el Pleistoceno. I Ciclos culturales. Sección de Geológicas, Zaragoza. Debates sobre la evolución (G. Meléndez, ed.). Publicaciones del Seminario de Paleontología de Zaragoza, Zaragoza: 35-46.

Carbonell, E.; Guilbaud, M.; Mora, R. (1983): Utilización de la lógica analítica para el estudio de tecno-complejos de cantos tallados. Cahier Noir, 1: 1-64.

Carbonell, E.; Guilbaud, M.; Mora, R. (1983): Utilización de la lógica analítica para el estudio de tecno-complejos de cantos tallados. Cahier Noir, 1: 1-64.

Carbonell, E.; Márquez, B.; Mosquera, M.; Ollé, A.; Rodríguez, X.P.; SAla, R.; Vergés, J.P. (1999): El Modo 2 en Galería. Análisis de la industria lítica y sus procesos técnicos. Atapuerca: ocupaciones humanas y paleoecología del yacimiento de Galería. (Antonio Rosas, Carlos Díez y E. Carbonell, eds.), Junta de Castilla y León, Valladolid (Memorias 7): 299-352.

Carbonell, E.; Mora, R. (1986): El sistema lógico-analitic i la teoría del "transfer". L'estudi dels complexes litics. Societat Catalana d'Arqueologia, Centre de Documentació, Dossier III. 
Carbonell, E.; Mosquera, M.; Ollé, A.; Rodríguez, X.P.; Sahnouni, M.; Sala, R.; Vergés, J.P. (2001): Structure morphotechnique de l'industrie lithique du Pléistocène infèrieur et moyen d'Atapuerca (Burgos, España). L'Anthropologie, 105: 259-280.

Carbonell, E.; Rodríguez, X.P.; Sala, R.; Vaquero, M. (eds.) (1992): New elements of the logical analytic system. First Internacional Meeting on Technical Systems to Configure Lithic Objets of scarce elaboration. Cahier Noir, 6, Tarragona.

Carbonell, E.; Sala, R. (1989): Cadena Operativa i “transfer” en els objectes d'ús humá i llur context. Empúries, 48-50: 176-183.

Carbonell, E.; Sala, R.; Barsky, D.; Celiberti, V. (2009): From Homogeneity to Multiplicity: a new approach to the study of archaic stone tools. Interdisciplinary approaches to the Oldowan (E. Hovers \& D. Braun eds.), Springer, Nueva York: 25-37

Chazan, M. (2009): Assessing the Lower to Middle Palaeolithic Transition. Sourcebook of Palaeolithic transitions (M. Champs y P.R. Champs, eds.), Springer, Nueva York: 237-244.

Clark, G. (1969): World Prehistory. A New Outline. Cambridge University Press, Cambridge.

Clark, J.G.D. (1952): Prehistoric Europe: The economic basis. Methuen, Londres.

Clarke, G.H. (2009): Accidents of History: Conceptual frameworks in Palaeoarchaeology. Sourcebook of Palaeolithic transitions (M. Champs y P.R. Champs, eds.), Springer, Nueva York: 19-42.

Clarke, D.L. (1984): Arqueología Analítica. Bellatera, Barcelona.

Cresswell, R. (1976): Techniques et culture, les bases d'un programme de travail. Techniques et culture, 1: 7-59.

DibBle, H. L. (1988): The interpretation of Middle Paleolithic scraper reduction patterns. L'Homme de Néandertal. Liége, E.R.A.U.L., 4: 49-58.

DibBLE, H. L. (1989): The implications of the stone tool types for the presence of language during the Lower and Middle Palaeolithic. The Human Revolution. Behavioural and Biological Perspectives in the Origins of Modern Humans. (P. Mellars y C. Stringer, eds), Edinburgh University Press.

Dibble, H. L. (1991): Mousterian assemblage variability on an interregional scale. Journal of Anthropological Research, 47(2): 239-257.

Dibble, H. L. (1995): Raw material availability, intensity of utilization, and Middle Paleolithic assemblage variability. The Middle Paleolithic site of Combe-Capelle Bas (France). (. H. L. Dibble y M. Lenoir, eds.), University Museum: University of Pennsylvania, Philadelphia: 289-315

DibBle, H. L.; Rolland, N. (1990): Beyond the Bordes-Binford debate: A synthesis of factors underlying assemblage variability in the Middle Paleolithic. New Perspectives of human adaptations and behabior in the Middle Paleolithic. (H. Dibble y P. Mellars, eds.), University Pennsylvania Press, Philadelphia.

Dibble, H. L.; Rolland, N. (1992): On assemblage variability in the Middle Paleolithic of Western Europe. History, perspectives, and a New Synthesis. The Middle Paleolithic: adaptation, behavior and variability. (H. L. Dibble y N. Rolland, eds.), University of Pennsylvania, Philadelphia: 1-28.

Dibble, H. L.; Roth, B; Lenoir, M. (1995): The use of raw material at Combe-Capelle Bas. The Middle Paleolithic site of Combe-Capelle Bas (France) (H. L. Dibble y M. Lenoir, eds.), University of Pennsylvania, Philadelphia: 259-287.

Díez Martín, F. (2004): La aplicación de los "Modos Tecnológicos” en el análisis de las industrias paleolíticas. Reflexiones desde la perspectiva Europea. SPAL, 12: 35-51.

Domínguez Rodrigo, M. (2008): Arqueología Neo-procesual: “Alive and kicking”. Algunas reflexiones desde el Paleolítico. Complutum, 19: 195-204.

FlanNery, K. (1967): Culture History vs. Culture Process: a debate in American archaeology. Scientific American, 217 (2): 119-122.

Gamble, C. (1990): El poblamiento paleolítico de Europa. Crítica, Barcelona.

GAmBle, C. (2001): Modes, movement and moderns. Quaternary International, 75: 5-10.

Geneste, J. M. (1985): Analyse lithique d'industries mousteriennes du Périgord: une approche technologique du comportement des groupes humains au Paléolithique moyen. Tesis Doctoral, inédita, Université de Bordeaux I.

Geneste, J. M. (1988a): Systèmes d'approvisionnement en matières premières au Paléolithique moyen et au Paléolithique supérieur en Aquitaine. L'Homme de Néandertal, 8, E.R.A.U.L., Liège: 61-70. 
Geneste, J. M. (1988b): Les industries de la Grotte Vaufrey: technologie du débitage, économie er circulation de la matière première lithique. La Grotte Vaufrey á Cenac et Saint-Julien (Dordogne). Paleoenvironnements, chronologie et activités humaines. (J. P. Rigaud, ed.), Mémoires de la Société Préhistorique Francaise. XIX, París.

Geneste, J. M. (1989): Economie des ressources lithiques dans le Moustérien du Sud-Ouest de la France. L'homme de Néandertal. Actes du Colloque International de Liège, 6, Liège: 75-97.

Geneste, J. M. (1990): Territoires de circulation au Paleólithique inférieur et moyen. Bulletin de la Société d'Anthropologie du Sud-Ouest, XXV(1): 13-30.

GenEsTe, J. M. (1991a). Systèmes techniques de production lithique: variations techo-économiques dans les processus de réalisation des outillages paléolithiques. Techniques et culture, 17-18: 1-35.

GENESTE, J. M. (1991b): L'approvisionnement en matières premières dans les systèmes de production lithique: la dimension spatiale de la technologie. Tecnología y cadenas operativas líticas (R. Mora, ed.), Barcelona: 1-35.

Groenen, M. (1994): Pour une histoire de la Préhistoire. Jérôme Million, Grenoble.

Hawley, A. (1991): Teoría de la Ecología humana. Tecnos, Madrid.

HAYDEN, B. (1989): From chopper to celt: the evolution of resharpening techniques. Time, energy and stone tools. (R. Torrence, ed.), Cambridge University Press, Cambridge: 7-16.

InIzAn, M.-L., Reduron, M., Roche, H. y Tixier, J. (1995): Technologie de la pierre taillée. CREP, París.

IsAAC, G. (1972): Chronology and the tempo of cultural change during the Pleistocene (W. Bishop y J. A. Miller, eds.), Scottish Academic Press, Edimburgo: 381-430.

IsAAC, G. (1976): Early hominids in action: a commentary of the contribution of archaeology to understanding the fossil record in East Africa. Yearbook of Physical Anthropology for 1975: 19-35.

IsAAC, G. (1981): Stone age visiting cards: approaches to the study of early land use patterns. Pattern of the Past. Studies in honour of David L. Clarke (G. Isaac \& H. Hodder, eds.), Cambridge: 31-156.

KuHN, S. L. (1991): Unpacking reduction: lithic raw material economy in the Mousterian of West-Central Italy. Journal of Anthropological Archaeology, 10: 76-106.

Kunn, S. L. (1992): On planning and curated technologies in the Middle Paleolithic. Journal of Anthropological Research, 48(3): 185-214.

KuHN, S. L. (1993): Mousterian technology as adaptative response: a case study. Hunting and animal exploitation in the Later Paleolithic and Mesolithic of Eurasia. (H. M. Bricker, P. Mellars y G.L. Peterkin, eds.), Archaeological Papers of the American Anthropological Association, 4: 25-31.

Kunn, S. L. (1995): Mousterian lithic technology. An ecological perspective. Princeton University Press.

LAPlaCe, G. (1956) : Typologie statistique et évolution des complexes à lames et lamelles. Bulletin de la Société Préhistorique Française, 54 (5-6): 271-290.

Laplace, G. (1958). Recherches sur l'origine et l'évolution des complexes leptolithiques. Quaternaria, V: 153240.

LAPLACE, G. (1972): La typologie analytique et structurale: base rationnelle d'étude des industries lithiques et osseuses. Banques de données archéologiques, Colloques nationaux du CNRS, 932, París: 91-142.

LEMONNIER, P. (1976): La description des chaines opératoires: contribution à l'analyse des systèmes techniques. Techniques et culture, 1: 100-151.

Leroi-Gourhan, A. (1964): Le geste et al parole. Technique et langage. Albin Michel, París.

Lieberman, D. E. (1993): The rise and fall of seasonal mobility among hunter-gatherers. The case of the Southern Levant. Current Anthropology, 34(5): 599-631.

Lyell, Charles. (1863): The geological evidences of the antiquity of man with remarks on the origin of species by variation. John Murray, Londres.

Lubbock, J. (1865): Prehistoric Times. Williams \& Norgate, Londres.

Maget, M. (1953): Guide d'étude directe des comportements culturels. Civilisations du Sud, París

Magne, M. P. (1989): Lithic reduction stages and assemblage formation processes. Experiments in lithic techno$\operatorname{logy}$ (D. S. Amick y R. P. Mauldin, eds.), BAR International Series, Oxford, 528: 15-31.

Mauss, M. (1947): Manuel d'Ethnographie. Payot, París.

Marks, A. E. (1988): The Middle to Upper Paleolithic transition in the Southern Levant: technological change as an adaptation to increasing mobility. L'Homme de Néandertal, E.R.A.U.L., Liège: 109-123. 
Meignen, L. (1988): Un exemple de comportement technologique différentiel selon les matières premières: Marillac, couches 9 et 10. L'Homme de Néandertal, E.R.A.U.L., Liège: 71-79.

Monnier, G. (2006) The Lower/Middle Palaeolithic periodization in Western Europe. Current Anthropology, 47: 709-729.

Mora, R., Martinez Moreno, J. y Terradas, X. (1991): Un proyecto de análisis: el Sistema Lógico Analítico (SLA). Tecnología y Cadenas Operativas Liticas. Treballs d'Arqueología: 173-199.

MoRTILLET, G. DE (1869) : Essai d'une classification des cavernes et des stations sous abri, fondée sur les produits de l'industrie humaine. Matériaux pour l'Histoire primitive et naturelle de l'Homme, cinquième année, $2^{\mathrm{a}}$ série, 3-4: 172-179.

Mortillet, G. DE (1872) : Classification de l'Âge de la pierre. Matériaux pour l'Histoire primitive et naturelle de l'Homme, huitième année, $2^{\mathrm{a}}$ série : 464-465.

Отте, M. (1990): L'occupation mousterienne de Sclayn (Belgique). EAZ Ethnogr.-Archäol. Z, 31: 78-101

Pelegrin, J. (1985): Reflétions sur le comportement technique. La signification culturelle des industries lithiques (M. Otte, ed.), Liège, Vol. 239: 72-88.

Pelegrin, J. (1990): Prehistoric lithic technology: some aspects of research. Archeological Review from Cambridge, 9, (1): 116-125.

Pelegrin, J. (1995): Technologie lithique. Le Châtelperronien de Roc-De-Combe (Lot) et de la Côte (Dordogne), Vol. 20, Cahiers du Quaternaire, París.

Rolland, N.; Dibble, H.L. (1990): A new synthesis of Middle Paleolithic variability. American Antiquity, 55(3): 480-499.

SÁnchez Yustos, P. (2009): El Paleolítico antiguo en la Cuenca del Duero. Instrumentos teóricos para el desarrollo de un modelo interpretativo de Arqueología económica. Tesis Doctoral inédita, Universidad de Valladolid

Sonneville-Bordes, D.; Perrot, J. (1954-1956): Lexique typologique du Paléolitique Supérieur. Bulletin de la Société Préhistorique Française, Tomos 51, 52 y 53.

Steward, J. (1950): Area research. Theory and practice. Social Science Research Council Bulletin, 63: 1-164

Steward, J. (1955) Theory of cultural Change. The methodology of multilinear evolution. University of Illinois Press, Urbana.

Strauss, L.G. (2007): Even the notion of a "Transitional Industry". New approaches to the study of Early Upper Paleolithic Transitional Industries in Western Eurasia (J. Riel Salvatore y G.A. Clark, eds.), British Archaeological Reports S-1620, Oxford: 11-18.

Strauss, L.G. (2009): Has the notion of "Transitions" in Paleolithic prehistory outlived its usefulness? The European record in wider context. Sourcebook of Palaeolithic transitions (M. Champs y P.R. Champs, eds.), Springer, Nueva York: 3-18.

Stringer, P.; Gamble, C. (1996): En busca de los Neandertales. Crítica, Barcelona.

Struever, S. (1971): Comments on archaeology data requirement and research strategies. American Antiquity, 36: 9-19.

Tavoso, A. (1984): Réflexion sur l'économie des matières premières au Moustérien. Bulletin de la Société d'Anthropologie du Sud-Ouest, 81(3): 79-82.

Tixier, J. (1982): Techniques de débitage: osons ne plus affirmer. Tailler! pour quoi faire: Préhistoire et technologie lithique (D. Cahen, ed.), Vol. 2, Studia Praehistorica Belgica: 13-22

TuRQ, A. (1986): Exploitation des matières premières lithiques et occupation du sol: 1'exemple du Moustérien entre Dorgogne et Lot. Relations entre les variations des paléo-milieux, le peuplement préhistorique et l'occupation $d u$ sol, Cahiers du Quaternaire, CNRS, París: 179-204.

TurQ, A. (1989): Approche technologique et économique du faciès Moustérien de type Quina: étude préliminaire. Bulletin de la Société Préhistorique Francaise, 86(8): 244-256.

TurQ, A. (1992): Raw material and technological studies of the Quina Mousterian in Perigord. The Middle Paleolithic: adaptation, behavior and variability. (H. Dibble y P. Mellars, eds.), University of Pennsylvania, Philadelphia: 75-85.

VAquero, M. (1997): Tecnología lítica y comportamiento humano: organización de las actividades técnicas y cambio diacrónico en el Paleolítico medio del Abric Romani (Capellades, Barcelona). Tesis Doctoral inédita, Universitat Rovira i Virgili, Tarragona. 
Vega Toscano, G. (2001): Aplicación de la metodología de los Programas de Investigación al análisis historiográfico del Paleolítico. Complutum, 12: 185-215.

Vega Toscano, G. (2003): La otra humanidad. La Europa de los Neandertales. Arco, Madrid.

Villa, P. (2001): Early Italy and the colonization of Western Europe. Quaternary International, 75: 113-130.

Villa, P. (2009): The Lower to Middle Paleolithic Transition. Sourcebook of Palaeolithic transitions (M. Champs y P.R. Champs, eds.), Springer, Nueva York: 265-272.

Willey, G. (1953): Archaeological theories and interpretation: The New World. Viking fund Publications in Anthropology, 2. 